\title{
Precision Medicine in Graves' Disease and Ophthalmopathy
}

\begin{abstract}
Giusy Elia ${ }^{1}$, Poupak Fallahi ${ }^{2 *}$, Francesca Ragusa ${ }^{3}$, Sabrina Rosaria Paparo ${ }^{3}$, Valeria Mazzi ${ }^{1}$, Salvatore Benvenga ${ }^{4,5,6}$, Alessandro Antonelli ${ }^{1}$ and Silvia Martina Ferrari ${ }^{3}$

${ }^{1}$ Department of Surgical, Medical and Molecular Pathology and Critical Area, University of Pisa, Pisa, Italy, ${ }^{2}$ Department of Translational Research and New Technologies in Medicine and Surgery, University of Pisa, Pisa, Italy, ${ }^{3}$ Department of Clinical and Experimental Medicine, University of Pisa, Pisa, Italy, ${ }^{4}$ Department of Clinical and Experimental Medicine, University of Messina, Messina, Italy, ${ }^{5}$ Master Program on Childhood, Adolescent and Women's Endocrine Health, University of Messina, Messina, Italy, ${ }^{6}$ Interdepartmental Program of Molecular and Clinical Endocrinology and Women's Endocrine Health, University Hospital, A.O.U. Policlinico G. Martino, Messina, Italy
\end{abstract}

Graves' disease (GD) is a condition caused by an autoimmune process involving the thyroid gland, whose main outcome is hyperthyroidism. TSAb start the autoimmune process stimulating the overproduction of thyroid hormones. In addition, TSAb can stimulate TSH-R expressed in fibroblasts and orbital pre-adipocytes leading to the manifestation of Graves' ophtalmopathy (GO). Also, autoantibodies directed against IGF-1R have an important role in immune-pathogenesis of GO. Fundamental is the role played by cytokines (IFN- $\gamma$, TNF- $\alpha, \|-6$ ), and Th1 chemokines in the immunepathogenesis of both disorders, particularly in the active phase. Novel discoveries in the field led to the investigation of promising therapies, such as immune-therapies towards specific antigens (for example against TSH-R), aiming in restoring the immune tolerance versus the immune dominant epitopes associated with autoimmunity in GD. Moreover, Etanercept (that blocks the TNF-mediated inflammatory responses), TCZ (that acts against the IL-6 receptor), and RTX (that acts against CD20) have proven to be useful and safe therapeutic options in refractory GO treatment. Furthermore, teprotumumab (a human monoclonal anti-IGF-1R blocking antibody), have been revealed effective in the treatment of patients with moderate-severe $\mathrm{GO}$ and it is now approved for GO therapy in United States. Molecules able to act as antagonists of CXCR3, or to block CXCL10, are also under study. More extensive researches are needed to deepen out these drugs as well as to identify new targeted and effective therapies, that will permit a more precise identification of $\mathrm{GD}$, or $\mathrm{GO}$, patients able to respond to specific targeted therapies.

Keywords: Graves' disease, Graves' ophthalmology, thyroid eye disease, teprotumumab, tocilizumab, rituximab, chemokine, cytokines

\section{INTRODUCTION}

The immune system has the important role to protect our body from foreign or inner attacks, but unfortunately this delicate mechanism can be broken and our immune system can attack the selfantigens leading to the appearance of autoimmune disorders. Several factors can contribute to this breakdown, such as environmental, genetics, immunological, hormonal conditions, being part of the "mosaic of autoimmunity" (Shoenfeld et al., 2019). Nowadays autoimmune disorders are largely widespread and in rising growth with women representing the mostly affected gender; moreover, 
autoimmune disorders might run together in the same person (Cooper and Stroehla, 2003; Lerner et al., 2015; Fallahi et al., 2019). The most frequent autoimmune disorders are the autoimmune thyroid disorders (AITD), directed against the thyroid gland, whose main clinical features are Graves' disease (GD), and Hashimoto's thyroiditis (HT) (Antonelli et al., 2015). Here we review the new pharmacological progresses made for the treatment of GD, and of Graves' ophtalmopathy (GO).

\section{Grave's Disease and Ophtalmopathy}

Graves' disease has a prevalence of about $1-1.5 \%$, in iodine sufficient West countries, with an incidence of 20-30 new cases $/ 100,000$ for year. The risk is higher for women, aged 35-55 years, and among African Americans (Smith and Hegedüs, 2016; Kahaly, 2020).

Several factors can predispose to the onset of GD, ranging from genetic, environmental, hormonal conditions to habits such as the smoke. Studies involving twins reinforced the role covered by genetic. A cohort study of 110,814 twins examined coaggregation and heritability of HT and GD. They observed a higher co-aggregation in monozygotic twins with respect to dizygotic twins, and found a high heritability for GD (Skov et al., 2020). Some variants of the DRB1, DQA1 and DQB1 genes of the human leukocyte antigen (HLA) class II genes are predictors of the development of GD, whereas others have a protective role, $H L A-D R B 1^{*} 07, H L A-C 03$, and $H L A-C^{\star} 16$ (Vejrazkova et al., 2018; Wémeau et al., 2018). Other immunecompetent genes whose variants may be involved in GD are PTPN22, CTLA4, CD40, FOXP3, ARID5, NRXN3, IKZF3. Also, other specific thyroid antigens such as "thyroid-stimulatinghormone receptor" (TSH-R), or Thyroglobulin (Tg), have been identified by a whole-genome linkage study as major AITD risk genes (Tomer et al., 2003; Vejrazkova et al., 2018). Susceptible individuals could be more easily influenced by environmental triggers, such as external radiation, iodine, selenium, smoking or viruses (Ferrari et al., 2017). Lately, 5 cases of GD reappearance, and a case of GO, after SARS COV-2 infection has been observed (Mateu-Salat et al., 2020; Harris and Al Mushref, 2021; JiménezBlanco et al., 2021; Lanzolla et al., 2021).

Therefore, these conditions predispose to the break of the immune tolerance towards thyroid antigens, mainly against the TSH-R. Anti-TSH-R autoantibodies (TRAb) are implicated in the thyroidal and extra-thyroidal manifestations of GD. TRAb are released by B lymphocytes, that infiltrate the thyroid gland during the autoimmune process. They are functionally divided in stimulating (TSAb), blocking (TBAb) and neutral antibodies, with the stimulating ones that induce the hyper-production of thyroid hormones, therefore leading to the clinical manifestations of hyperthyroidism (Smith and Hegedüs, 2016; Antonelli et al., 2020). TSAb have a significant role not only in the thyroid gland, but also in the extra-thyroidal manifestations of GD, such as GO, or pretibial myxedema. Other thyroid antigens are involved in the autoimmune process of GD, such as thyroid peroxydase (TPO) and/or Tg, whose antibodies are found in about $50-70 \%$ of cases of GD (Wémeau et al., 2018). The involvement of autoantibodies binding the insulin-like growth factor-1 receptor (IGF-1R) has been found to be implicated in the development of GO (Smith,
2019). They are able to induce the expression of the chemokine "regulated on activation normal T cell expressed and secreted" (RANTES) and IL-16 (Pritchard et al., 2002; Pritchard et al., 2003; Smith, 2019) attracting T lymphocytes, that enter into the site of tissue damage inducing and perpetuating the inflammatory process (Cruikshank et al., 1987; Schall et al., 1988; Smith, 2019).

\section{Cytokines/Chemokines in GD}

Fundamental is also the role covered by "Th1 chemokines" (CXCL10, CXCL9, CXCL11), and their (C-X-C)R3 receptor in the immune-pathogenesis of both disorders. In the active phase of GD prevails a Th1 immune response, in which, subsequently to a CXCL10 production by resident follicular epithelial cells, occurs a recruitment of Th1 cells. This process leads to the initiation, and amplification of the inflammation (Romagnani et al., 2002; Antonelli et al., 2020) (Table 1).

In basal condition thyroid follicular cells do not secrete Th1 chemokines, while a release occurs under interferon (IFN)- $\gamma$, and it is higher under a combined IFN- $\gamma$ and tumor necrosis factor (TNF)- $\alpha$ (IFN- $\gamma+$ TNF- $\alpha$ ) stimulation (Antonelli et al., 2006a; Antonelli et al., 2009; Antonelli et al., 2010; Ferrari et al., 2015; Fallahi et al., 2020). Therefore, the cytokines stimulation made thyrocytes largely involved in the inflammatory process through the release of Th1 chemokine. The peroxisome proliferatoractivated receptor (PPAR)- $\gamma$ agonists, such as PPAR- $\alpha$ agonists, instead inhibit this process (Antonelli et al., 2009; Antonelli et al., 2010; Antonelli et al., 2011; Ferrari et al., 2015).

Both the active and the relapse phase of GD is characterized by high circulating Th1 chemokines, that decline with methimazole (MMI) therapy. The immune-modulatory effect of MMI is associated with the decrease of serum CXCL10, that achieves normal levels with thyroid hormones normalization, or with GD in remission. The reduction of circulating CXCL10 was not associated with the reduction of AbTg or AbTPO levels, but with the decrease of TRAb (Antonelli et al., 2006a; Antonelli et al., 2006b; Antonelli et al., 2013).

CXCL10 serum levels were also assessed in GD patients who underwent total thyroidectomy or radioactive iodine (RAI) treatment. The decrease of CXCL10 levels following these treatments suggests that the site of production of this chemokine is the thyroid gland itself (Antonelli et al., 2006c; Antonelli et al., 2007; Leite et al., 2011).

Furthermore, a study investigated CXCL10 levels in subjects with: 1) 16 new diagnoses of GD in therapy with MMI; 2) 15 relapsed GD in treatment with RAI; 3) 18 controls. Subjects treated with MMI reported a decline of CXCL10 and euthyroidism after 6 and 12 months; those treated with RAI showed a reduction of CXCL10 levels after 3, 6, 9, and 12 months, with a similar TRAb decrease (Leite et al., 2011).

\section{Cytokines/Chemokines in GO}

The onset of GO and GD are often concomitant, with GO involving almost $30-50 \%$ of GD patients. Subjects who are more prone to develop a GO are smoker, or patients with a severe hyperthyroidism, and those with very high levels of TSAb. Athough a primary prevention of GO is not available, the progression from a subclinical condition into overt and/or 
TABLE 1 | Cytokines and/or Chemokines in Graves' disease and in Graves' Ophtalmopathy.

Cytokines and/or chemokines

IL-6

$\mathrm{TNF}-\alpha / \mathrm{IFN}-\gamma$

CXCL10/CXCL9

Studies [ref]

-sIL-6R concentrations were higher in GD patients with active inflammatory thyroid-associated ophthalmopathy than those in patients with inactive or absent thyroid-associated ophthalmopathy Salvi et al. (1996)

-IFN- $\gamma$, or IFN- $\gamma+$ TNF- $\alpha$ combination stimulate Th1 chemokines in TFCs Antonelli et al. (2006a); Antonelli et al. (2009); Antonelli et al. (2010); Ferrari et al. (2015); Fallahi et al. (2020)

-IFN- $\gamma$, or IFN- $\gamma+$ TNF- $\alpha$ combination stimulate Th1 chemokines in the primary cell cultures of retro-bulbar cells of GO patients Antonelli et al. (2006a); Dong et al. (2011)

-Maximal expression of CXCL10 and CXCL9 was found in the thyroid gland of patients with recent-onset GD and was correlated with IFN- $\gamma$. High levels of CXCL10 could be measured in the serum of patients with short-duration GD Romagnani et al. (2002)

-Significant reductions in CXCL9 and CXCL10 serum concentrations during CS and TR treatment as compared both to control group and to basal values in GO patients Mysliwiec et al. (2012)

CXCL10

-Thyrocytes and retrobulbar cell types participate in the self-perpetuation of inflammation by releasing chemokines under the influence of cytokines. PPAR- $\gamma$ activation plays an inhibitory role in this process Antonelli et al. (2006a)

-sCXCL10 are associated with the active phase of GD in both newly diagnosed and relapsing hyperthyroid patients. The reduction of SCXCL10 in treated patients with GD may be related to the immunomodulatory effects of MMl Antonelli et al, (2006b)

- sCXCL10 are higher in newly diagnosed hyperthyroid patients with GD than in those with TNG, and decrease when euthyroidism is achieved with antithyroid therapy Antonelli et al. (2006c)

- High sCXCL10 are associated with the hyperthyroid phase in GD but not TNG Antonelli et al. (2007)

- Data show a relationship between serum CXCL10 and GD activity Leite et al. (2011)

-CXCL10 participates in the early inflammatory response after radioactive iodine therapy in patients with GD and shows a strong association with the autoimmune process Dong et al., 2011

CXCL9/CXCL11

-Thyrocytes and retrobulbar cell types from patients with GD and GO released CXCL9 and CXCL11 chemokines when stimulated with cytokines. PPAR- $\gamma$ activation plays an inhibitory role in this process Antonelli et al. (2009)

- PPAR- $\alpha$ has been found in GD and control thyrocytes. PPAR- $\alpha$ activators are potent inhibitors of the secretion of CXCL9 and CXCL11 Antonelli et al. (2010)

-Serum CXCL9 and CXCL11 levels are associated with the active phase of GD both in newly diagnosed and relapsing hyperthyroid patients. The reduction of serum CXCL9 and CXCL11 levels in GD patients in treatment with MMI, may be related to the immunomodulatory effects of MMI Antonelli et al. (2013)

-PPAR- $\alpha$ activators inhibit CXCL9 and CXCL11 chemokines in normal and GO fibroblasts and preadipocytes Antonelli et al. (2012).

CXCL10/CCL2

-CCL2 is modulated by IFN- $\gamma$ and TNF- $\alpha$ in GD and normal thyrocytes. PPAR- $\alpha$ activators inhibit the secretion of CXCL10 and CCL2 in thyrocytes Antonelli et al. (2011)

-EOM participates in the self-perpetuation of inflammation by releasing CXCL10 and CCL2 chemokines under the influence of cytokines, in GO. PPAR- $\gamma$ agonist activation plays an inhibitory role on CXCL10, but stimulates the release of

CCL2 Antonelli et al. (2014)

CS, Corticosteroids; EOM, extra-ocular muscle; GD, Graves' disease; GO, Graves' Ophtalmopathy; IFN, Interferon; IL, interleukin; MMI, methimazole; PPAR, peroxisome proliferatoractivated receptor; sCXCL10, Serum levels of CXCL10; TFCs, thyroid follicular cells; TNG, toxic nodular goitre; TR, teleradiotherapy.

severe ones can be avoided through an early diagnosis, an accurate control of thyroid function, stop of smoking, and with the early therapy of mild GO (Wiersinga and Bartalena, 2002; Perricone et al., 2016; Smith et al., 2017) (Table 1).

GO retro-bulbar cells (fibroblasts and preadipocytes) are highly involved in the perpetuation of the orbital inflammation by releasing Th1 chemokines (CXCL10, CXL11, CXCL9) under the influence of IFN- $\gamma$. Higher serum CXCL10 levels have been observed in GO patients with active disease in comparison to the inactive ones. Moreover, Th1 chemokines were basally absent in the primary cell cultures of retro-bulbar cells of GO patients; whereas their release was stimulated by IFN- $\gamma$, or IFN- $\gamma+$ TNF- $\alpha$ stimulation (Antonelli et al., 2006a; Dong et al., 2011). PPAR- $\alpha,-\delta$, and $-\gamma$ are found in GO fibroblasts or preadipocytes, and the PPAR- $\gamma$ agonists showed an inhibitory role on Th1 chemokines release (Antonelli et al., 2009; Antonelli et al., 2012).
Another study explored the involvement of retro-bulbar myoblasts in the immune-pathogenesis of GO (Antonelli et al., 2014). High serum CXCL10 levels have been observed in both patients having active GO associated with extraocular muscle (EOM) or with orbital fat involvement, in comparison with controls. CXCL10 was not detectable in primary EOM cells from GO patients, whereas it was released under the cytokines stimulation (IFN- $\gamma$ and/or TNF- $\alpha$ ). Therefore, EOM are involved in the inflammatory GO process through the release of Th1 chemokines (Antonelli et al., 2014).

A potential use of Th1 chemokines as markers of GO activity has been investigated by a study that involved forty-two GO subjects of which: 20 were GD patients (half in euthyroidism and half in hyperthyroidism); 15 GO patients in euthyroidism [previously treated with intravenous of methylprednisolone (ivMP) and teleradiotherapy], and seven were controls. Interestingly, a significant decrease of Th1 chemokines 
occurred after ivMP and teleradiotherapy. The reduction of circulating Th1 chemokines was not associated with the reduction of AbTg or AbTPO levels, nor of TRAb.

Therefore, these chemokines may aid in the therapeutic decision-making of GO patients (Mysliwiec et al., 2012).

\section{THERAPY FOR GD}

\section{Antithyroid Drugs}

MMI, carbimazole, and propylthiouracil (PTU) are the firstchoice therapy for GD. These drugs act by inhibiting TPO, and blocking the synthesis of thyroid hormones. PTU also blocks extrathyroidal deiodination of T4 to T3. The toxicity profile of these drugs makes them the preferred with respect to radioiodine (Bartalena et al., 2016; Burch and Cooper, 2015; Antonelli et al., 2020), however the risk of relapse after therapies is high. Furthermore, MMI and PTU have immune-modulatory effect reducing TSAb levels (Antonelli et al., 2013).

\section{Radioiodine Therapy}

RAI has been widely used; it gives relief from symptoms of hyperthyroidism within weeks. Antithyroid drugs can be suspended 3-7 days before and after radioiodine in order to improve its effectiveness. However, radioiodine can cause or worsen GO. Therefore, a close monitoring of the thyroid function should be performed, and when hypothyroidism occurs, it needs to be treated as soon as possible (Galetta et al., 2008; Smith and Hegedüs, 2016).

\section{Surgery}

Surgery is needed in particular conditions, such as if the patient do not want to receive anti-thyroid drugs, or radioiodine; in presence of a large goiter; and for women who would like to have pregnancy. The patients must reach euthyroidism, before they can undergo surgery. This will reduce the risk of complications (Feroci et al., 2014; Smith and Hegedüs, 2016).

\section{Antigen-specific Immunotherapy}

The antigen-specific immunotherapies aim to re-establish an immunological tolerance against the immune dominant epitopes involved in autoimmunity, without inducing generalized immunosuppression (Pearce et al., 2019). A study investigated a combination of two TSHR peptides (ATX-GD-59) in 12 subjects with mild-to-moderate untreated hyperthyroidism. A potential efficacy of this treatment has been suggested; $70 \%$ of the treated subjects reported an improvement in free thyroid hormones (Pearce et al., 2019).

\section{THERAPY FOR GO}

\section{Corticosteroids Therapy}

The common treatment for active GO are high-dose of ivMP. A multicenter trial demonstrated the effectiveness of ivMP in improving inflammation in about $80-70 \%$ of the cases, and eye muscle function in $50 \%$. Nevertheless, about $20 \%$ were no significantly responders to the treatment, and progression disease or compression of the optic nerve occurred in about $4 \%$ of the subjects (Bartalena et al., 2012).

Therefore, new targets involved in the autoimmune reaction have been taken in accounts for the development of new drugs, such as TSH-R, the IGF-1R (on fibroblasts), T or B lymphocytes, chemokines and cytokines (Fallahi et al., 2016).

\section{TSH-R Antagonists}

Drugs acting against TSH-R have been recently investigated. Promising results have been obtained by a molecule NCGC0022960 able to reduce the production of hyaluronic acid in primary cell culture of retro-orbital fibroblasts/ adipocytes of GO (Emerson, 2011; Turcu et al., 2013).

In a patients with follicular thyroid cancer (FTC), GD, with high levels of TSAb, and severe GO, was tested K1-70 a monoclonal antibody anti-TSHR. After the start of the therapy, the TSAb activity decreased and GO improved. Moreover, on K1-70 monotherapy during the pause in lenvatinib, used for the treatment of FTC, occurs a stabilization of several metastatic lesions (Ryder et al., 2021).

\section{Etanercept and Tocilizumab}

Cytokines are largely involved in the autoimmune process of the GO. TNF- $\alpha$ and IL- 6 have a crucial role in this process (Bahn, 2010).

Etanercept is a dimeric protein able to bind two molecules of TNF, avoiding its interaction with receptors on the cell surface, and subsequently the TNF-mediated inflammatory responses. This molecule is the choice option for different autoimmune disorders [e.g. rheumatoid arthritis (RA), ankylosing spondylitis in adults, and juvenile idiopathic arthritis or plaque psoriasis in paediatric patients] (Scott, 2014). In a pilot study the efficacy of etanercept was investigated in $10 \mathrm{GO}$ subjects $(25 \mathrm{mg}$ twice weekly, were administered for 12 weeks). An improvement was observed in $60 \%$ of patients; a reactivation of GO occurred in three patients after cessation. No serious adverse events (AEs) or side effects were registered during a follow-up of 18 months (Paridaens et al., 2005). Another paper reported a case of a patient with RA and GO. She was treated with etanercept for RA achieving also a clinical improvement of GO symptoms (Boskovic et al., 2019). Additional researches are needed in order to evaluate the effectiveness of TNF- $\alpha$ inhibitors, and to compare its side effects with the current medical treatment.

The cytokine IL-6 is released by $\mathrm{T}$ lymphocytes and macrophages and has a pro-inflammatory activity. GO patients in the active phase showed increased levels of circulating IL-6 and of its receptor (Salvi et al., 1996). The monoclonal antibody (mAb) tocilizumab (TCZ) acts against the IL- 6 receptor, and received the approval for the treatment of RA, systemic juvenile idiopathic arthritis and Castleman's disease (Emery et al., 2008; Yokota et al., 2008). TCZ was evaluated in $18 \mathrm{GO}$ patients, not responders to corticosteroids (CS). Thirteen patients showed a decreased proptosis, fifteen had an improvement of the extraocular motility and seven out of 13 resolved their diplopia (Pérez-Moreiras et al., 2014). Another open-label multicenter study assessed the effectiveness of TCZ 
enrolling 48 patients with glucocorticoid-resistant GO (SánchezBilbao et al., 2020). The follow-up lasts for a mean of $16.1 \pm$ 2.1 months, and it was observed a decrease of disease activity [Clinical Activity Score (CAS) $\leq 3$ ] in many patients; TCZ was withdrawn in 29 cases, because of low disease activity in 25 cases, or inefficacy in four subjects. No serious AEs were registered. Thereby, TCZ appears an efficacy, useful and safe therapeutic option in refractory GO treatment (Sánchez-Bilbao et al., 2020).

\section{Rituximab}

Rituximab (RTX) acts against CD20 placed on B cells; thereby it induces $\mathrm{B}$ cells death, and is indicated in the therapy of those diseases characterized by elevated levels of B-lymphocytes or dysfunctional B-lymphocytes, and overactive B-cells. This mAb has no effect on plasma cells, it doesn't interfere with the antibody synthesis (Ahuja et al., 2008). Since RTX reduces the number of B lymphocytes, the burden of cytokines and the secreted autoantibodies, it has been suggested for the treatment of GO (Salvi et al., 2012). Conflicting results were reported about the efficacy of RTX in GO.

A study included $25 \mathrm{GO}$ subjects in a prospective, placebocontrolled, randomized trial (Stan et al., 2015); patients received two RTX infusions, or two saline infusions, 2 weeks apart. RTX appeared not effective in GO, because no differences were registered about the improvement of CAS with respect to placebo (Stan et al., 2015).

However, another double-blind, randomized trial enrolling 32 subjects reported different outcomes. The patients received RTX or ivMP; $100 \%$ of RTX patients achieved an improvement at 24 weeks, compared to 69\% after ivMP, therefore assessing a higher efficacy of RTX than ivMP in GO patients (Salvi et al., 2015).

Recently a multicenter retrospective study (Deltour et al., 2020) investigated the efficacy of RTX in forty GO patients resistant to CS, or in cases of CS dependence. The Authors found that RTX is effective as a second-line treatment of these patients, especially if the disease is recent and active; and when it is administered in the early phase of the disease. The time of administration may explain the contradictory results obtained in the previous randomized studies (Salvi et al., 2015; Stan et al., 2015; Deltour et al., 2020).

\section{Teprotumumab}

An overexpression of IGF-1R has been found in orbital connective tissues, $\mathrm{T}$ and $\mathrm{B}$ cells in GD and GO. GD patients generated autoantibodies that are able to bind to IGF-1R and initiate the signaling from the TSHR/IGF-1R physical and functional protein complex. Therefore, the use of mAbs against IGF-1R may attenuate signaling from either TSHR or IGF-1R (Smith, 2021).

Teprotumumab (RV 001, R1507) is a human monoclonal antiIGF-1R blocking antibody. An in vitro study, showed its efficacy in reducing the fibrocyte display of IGF-1R and TSH-R, such as their downstream signals, blocking the induction of proinflammatory cytokines (Chen et al., 2014).

A first multicenter, double-masked, randomized, placebocontrolled trial was carried out to investigate the efficacy of teprotumumab in patients with active, moderate-to-severe ophthalmopathy (Smith et al., 2017). The 88 enrolled subjects were randomly assigned to the placebo group or to the teprotumumab group. $69 \%$ of patients of the teprotumumab group had a response at week $24(p<0.001)$, with respect to the $20 \%$ of the placebo group. Moreover, the response was rapidly achieved $(p<0.001)$ in the teprotumumab group, $43 \%$ at week 6 , against only $4 \%$ of the placebo. These findings supported the efficacy of this drug in reducing proptosis and CAS in patients with active GO (Smith et al., 2017).

A subsequent randomized, double-masked, placebocontrolled, phase 3, multicenter trial, involved 83 patients with moderate to severe GO (with a duration of GO $<9$ months), of whom 41 received teprotumumab and 42 placebo. Teprotumumab led to better outcomes in proptosis, CAS, diplopia, and quality of life than placebo; serious AEs were uncommon (Douglas et al., 2020). Additionaly, the non responders were included in an extension of the phase 3 trial; they received teprotumumab as an open label, regardless of whether or not they had received the active drug, or placebo, during the 24-weeks treatment phase (Smith, 2021). The response to the therapy occurs in a similar fraction of patients, such as in the initial intervention. Follow-up data (of phase 3 trial, plus extension study) revealed that the majority of patients who responded with amelioration of proptosis and diplopia at week 24 maintained their responses (56 and 58\%, respectively) (Smith, 2021).

These studies showed the efficacy of teprotumumab as well as its safety. The AEs encountered were mild to moderate in severity, with the most common being hyperglycemia found especially in patients with diabetes, and easily managed by adjusting the therapy. Other AEs were muscle cramps, hearing abnormalities, hair loss, diarrhea and dysgeusia (Chen et al., 2014; Smith et al., 2017; Smith, 2021).

Teprotumumab has been approved by the US FDA for the therapy of GO, and is now in clinical use in North America (Smith, 2021).

However, more studies are needed to assess its effectiveness in such conditions e.g. patients with a chronic and less active GO condition or with an impaired vision due to compressive optic neuropathy (Markham, 2020; Smith, 2021).

\section{CONCLUSION}

Graves' disease (GD) is a condition caused by an autoimmune process involving the thyroid gland, whose main outcome is hyperthyroidism. TSAb start the autoimmune process stimulating the overproduction of thyroid hormones. In addition, TSAb can stimulate TSH-R expressed in fibroblasts and orbital pre-adipocytes, leading to the manifestation of GO. Also, autoantibodies directed against IGF-1R have an important role in immune-pathogenesis of GO. Fundamental is the role played by cytokines (IFN- $\gamma$, TNF- $\alpha$, Il-6), and Th 1 chemokines in the immune-pathogenesis of both disorders, particularly in the active phase.

Novel discoveries (Table 2) in the field led to the investigation of promising therapies, such as immune-therapies towards 
TABLE 2 | Latest drugs for Graves' Ophtalmopathy.

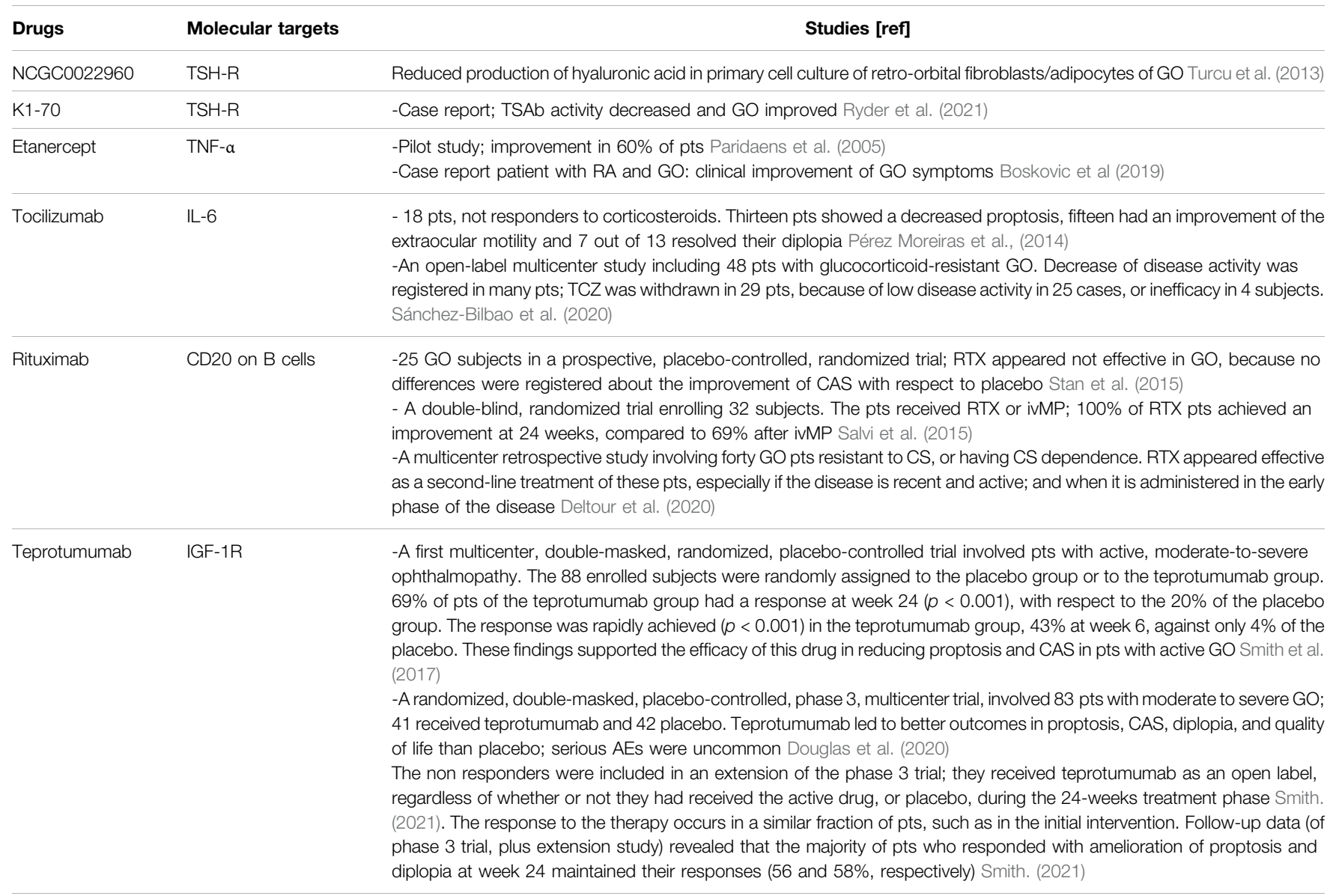

AEs, Adverse Events; CAS, Clinical Activity Score; CS, Corticosteroids; GO, Graves' Ophtalmopathy; ivMP, Intravenous Methylprednisolone; Pts, Patients; RA, Rheumatoid Arthritis; RTX, rituximab; TCZ, tocilizumab; TNF, Tumor Necrosis Factor TSAb, Thyroid Stimulating Antibodies; TSH-R, Thyroid-stimulating-hormone Receptor.

specific antigens (for example against TSH-R), aiming in restoring the immune tolerance in GD.

After the initial attempt of biologic therapies in GO (Antonelli et al., 1992; Baschieri et al., 1997), more recently, etanercept (that blocks the TNF-mediated inflammatory responses), TCZ (that acts against the IL-6 receptor), and RTX (that acts against CD20) have proven to be useful and safe therapeutic options in refractory GO treatment. Furthermore, teprotumumab (a human monoclonal antiIGF-1R blocking antibody), has been revealed effective in the treatment of patients with moderate-severe GO and it is now approved for GO therapy in United States.

\section{REFERENCES}

Ahuja, A., Anderson, S. M., Khalil, A., and Shlomchik, M. J. (2008). Maintenance of the Plasma Cell Pool Is Independent of Memory B Cells. Proc. Natl. Acad. Sci. U S A. 105, 4802-4807. doi:10.1073/pnas.0800555105

Antonelli, A., Fallahi, P., Elia, G., Ragusa, F., Paparo, S. R., Ruffilli, I., et al. (2020). Graves' Disease: Clinical Manifestations, Immune Pathogenesis (Cytokines and Chemokines) and Therapy. Best Pract. Res. Clin. Endocrinol. Metab. 34, 101388. doi:10.1016/j.beem.2020.101388
More, extensive researches are needed to deepen out these drugs as well as to identify new targeted and effective therapies, that will permit a more precise identification of $\mathrm{GD}$, or GO, patients able to respond to specific targeted therapies.

\section{AUTHOR CONTRIBUTIONS}

GE, PF, AA and SMF conceived the paper. All authors reviewed and approved the final version of the manuscript.

Antonelli, A., Fallahi, P., Rotondi, M., Ferrari, S. M., Serio, M., and Miccoli, P. (2006c). Serum Levels of the Interferon-Gamma-Inducible Alpha Chemokine CXCL10 in Patients with Active Graves' Disease, and Modulation by Methimazole Therapy and Thyroidectomy. Br. J. Surg. 93, 1226-1231. doi:10.1002/bjs.5401

Antonelli, A., Ferrari, S. M., Corrado, A., Di Domenicantonio, A., and Fallahi, P. (2015). Autoimmune Thyroid Disorders. Autoimmun. Rev. 14, 174-180. doi:10.1016/j.autrev.2014.10.016

Antonelli, A., Ferrari, S. M., Corrado, A., Ferrannini, E., and Fallahi, P. (2013). Increase of Interferon- $\gamma$ Inducible CXCL9 and CXCL11 Serum Levels in 
Patients with Active Graves' Disease and Modulation by Methimazole Therapy. Thyroid 23, 1461-1469. doi:10.1089/thy.2012.0485

Antonelli, A., Ferrari, S. M., Corrado, A., Franceschini, S. S., Gelmini, S., Ferrannini, E., et al. (2014). Extra-ocular Muscle Cells from Patients with Graves' Ophthalmopathy Secrete $\alpha$ (CXCL10) and $\beta$ (CCL2) Chemokines under the Influence of Cytokines that Are Modulated by PPAR $\gamma$. Autoimmun. Rev. 13, 1160-1166. doi:10.1016/j.autrev.2014.08.025

Antonelli, A., Ferrari, S. M., Fallahi, P., Frascerra, S., Santini, E., Franceschini, S. S., et al. (2009). Monokine Induced by Interferon Gamma (IFNgamma) (CXCL9) and IFNgamma Inducible T-Cell Alpha-Chemoattractant (CXCL11) Involvement in Graves' Disease and Ophthalmopathy: Modulation by Peroxisome Proliferator-Activated Receptor-Gamma Agonists. J. Clin. Endocrinol. Metab. 94, 1803-1809. doi:10.1210/jc.2008-2450

Antonelli, A., Ferrari, S. M., Frascerra, S., Corrado, A., Pupilli, C., Bernini, G., et al. (2011). Peroxisome Proliferator-Activated Receptor a Agonists Modulate Th1 and Th2 Chemokine Secretion in normal Thyrocytes and Graves' Disease. Exp. Cel Res 317, 1527-1533. doi:10.1016/j.yexcr.2011.04.007

Antonelli, A., Ferrari, S. M., Frascerra, S., Pupilli, C., Mancusi, C., Metelli, M. R., et al. (2010). CXCL9 and CXCL11 Chemokines Modulation by Peroxisome Proliferator-Activated Receptor-Alpha Agonists Secretion in Graves' and normal Thyrocytes. J. Clin. Endocrinol. Metab. 95, E413-E420. doi:10.1210/ jc.2010-0923

Antonelli, A., Ferrari, S. M., Frascerra, S., Ruffilli, I., Gelmini, S., Minuto, M., et al. (2012). Peroxisome Proliferator-Activated Receptor- $\alpha$ Agonists Modulate CXCL9 and CXCL11 Chemokines in Graves' Ophthalmopathy Fibroblasts and Preadipocytes. Mol. Cel Endocrinol 349, 255-261. doi:10.1016/ j.mce.2011.11.001

Antonelli, A., Rotondi, M., Fallahi, P., Grosso, M., Boni, G., Ferrari, S. M., et al. (2007). Iodine-131 Given for Therapeutic Purposes Modulates Differently Interferon-Gamma-Inducible Alpha-Chemokine CXCL10 Serum Levels in Patients with Active Graves' Disease or Toxic Nodular Goiter. J. Clin. Endocrinol. Metab. 92, 1485-1490. doi:10.1210/jc.2006-1571

Antonelli, A., Rotondi, M., Fallahi, P., Romagnani, P., Ferrari, S. M., Barani, L., et al. (2006b). Increase of Interferon-Gamma-Inducible CXC Chemokine CXCL10 Serum Levels in Patients with Active Graves' Disease, and Modulation by Methimazole Therapy. Clin. Endocrinol. (Oxf) 64, 189-195. doi:10.1111/j.13652265.2006.02447.x

Antonelli, A., Rotondi, M., Ferrari, S. M., Fallahi, P., Romagnani, P., Franceschini, S. S., et al. (2006a). Interferon-gamma-inducible Alpha-Chemokine CXCL10 Involvement in Graves' Ophthalmopathy: Modulation by Peroxisome Proliferator-Activated Receptor-Gamma Agonists. J. Clin. Endocrinol. Metab. 91, 614-620. doi:10.1210/jc.2005-1689

Antonelli, A., Saracino, A., Alberti, B., Canapicchi, R., Cartei, F., Lepri, A., et al. (1992). High-dose Intravenous Immunoglobulin Treatment in Graves' Ophthalmopathy. Acta Endocrinol. (Copenh) 126, 13-23. doi:10.1530/ acta. 0.1260013

Bahn, R. S. (2010). Graves' Ophthalmopathy. N. Engl. J. Med. 362, 726-738. doi:10.1056/NEJMra0905750

Bartalena, L., Burch, H. B., Burman, K. D., and Kahaly, G. J. (2016). A 2013 European Survey of Clinical Practice Patterns in the Management of Graves' Disease. Clin. Endocrinol. (Oxf) 84, 115-120. doi:10.1111/cen.12688

Bartalena, L., Krassas, G. E., Wiersinga, W., Marcocci, C., Salvi, M., Daumerie, C., et al. (2012). Efficacy and Safety of Three Different Cumulative Doses of Intravenous Methylprednisolone for Moderate to Severe and Active Graves' Orbitopathy. J. Clin. Endocrinol. Metab. 97, 4454-4463. doi:10.1210/jc.20122389

Baschieri, L., Antonelli, A., Nardi, S., Alberti, B., Lepri, A., Canapicchi, R., et al. (1997). Intravenous Immunoglobulin versus Corticosteroid in Treatment of Graves' Ophthalmopathy. Thyroid 7, 579-585. doi:10.1089/thy.1997.7.579

Boskovic, O., Medenica, S., Radojevic, N., and Zarkovic, M. (2019). Etanercept in the Treatment of Graves' Ophthalmopathy with Primary Hypothyroidism and Rheumatoid Arthritis. Cent. Eur. J. Immunol. 44, 463-465. doi:10.5114/ ceji.2019.92803

Burch, H. B., and Cooper, D. S. (2015). Management of Graves Disease: A Review. JAMA 314, 2544-2554. doi:10.1001/jama.2015.16535

Chen, H., Mester, T., Raychaudhuri, N., Kauh, C. Y., Gupta, S., Smith, T. J., et al. (2014). Teprotumumab, an IGF-1R Blocking Monoclonal Antibody Inhibits
TSH and IGF-1 Action in Fibrocytes. J. Clin. Endocrinol. Metab. 99, E1635-E1640. doi:10.1210/jc.2014-1580

Cooper, G. S., and Stroehla, B. C. (2003). The Epidemiology of Autoimmune Diseases. Autoimmun. Rev. 2, 119-125. doi:10.1016/s1568-9972(03)00006-5

Cruikshank, W. W., Berman, J. S., Theodore, A. C., Bernardo, J., and Center, D. M. (1987). Lymphokine Activation of T4+ T Lymphocytes and Monocytes. J. Immunol. 138, 3817-3823.

Deltour, J. B., d'Assigny Flamen, M., Ladsous, M., Giovansili, L., Cariou, B., Caron, P., et al. (2020). Efficacy of Rituximab in Patients with Graves' Orbitopathy: a Retrospective Multicenter Nationwide Study. Graefes Arch. Clin. Exp. Ophthalmol. 258, 2013-2021. doi:10.1007/s00417-02004651-6

Dong, Q. Y., Li, S. J., Gao, G. Q., Liu, X. M., Li, W. X., Liang, C. G., et al. (2011). Short-term Effect of Radioactive Iodine Therapy on CXCL-10 Production in Graves' Disease. Clin. Invest. Med. 34, E262. doi:10.25011/cim.v34i5.15668

Douglas, R. S., Kahaly, G. J., Patel, A., Sile, S., Thompson, E. H. Z., Perdok, R., et al. (2020). Teprotumumab for the Treatment of Active Thyroid Eye Disease. $N$. Engl. J. Med. 382, 341-352. doi:10.1056/NEJMoa1910434

Emerson, C. H. (2011). When Will Thyrotropin Receptor Antagonists and Inverse Thyrotropin Receptor Agonists Become Available for Clinical Use? Thyroid 21, 817-819. doi:10.1089/thy.2011.2108

Emery, P., Keystone, E., Tony, H. P., Cantagrel, A., van Vollenhoven, R., Sanchez, A., et al. (2008). IL-6 Receptor Inhibition with Tocilizumab Improves Treatment Outcomes in Patients with Rheumatoid Arthritis Refractory to Anti-tumour Necrosis Factor Biologicals: Results from a 24-week Multicentre Randomised Placebo-Controlled Trial. Ann. Rheum. Dis. 67, 1516-1523. doi:10.1136/ard.2008.092932

Fallahi, P., Elia, G., Ragusa, F., Ruffilli, I., Camastra, S., Giusti, C., et al. (2019). The Aggregation between AITD with Rheumatologic, or Dermatologic, Autoimmune Diseases. Best Pract. Res. Clin. Endocrinol. Metab. 33, 101372. doi:10.1016/j.beem.2019.101372

Fallahi, P., Ferrari, S. M., Elia, G., Nasini, F., Colaci, M., Giuggioli, D., et al. (2016). Novel Therapies for Thyroid Autoimmune Diseases. Expert Rev. Clin. Pharmacol. 9, 853-861. doi:10.1586/17512433.2016.1157468

Fallahi, P., Ferrari, S. M., Ragusa, F., Ruffilli, I., Elia, G., Paparo, S. R., et al. (2020). Th1 Chemokines in Autoimmune Endocrine Disorders. J. Clin. Endocrinol. Metab. 105, 1046-1060. doi:10.1210/clinem/dgz289

Feroci, F., Rettori, M., Borrelli, A., Coppola, A., Castagnoli, A., Perigli, G., et al. (2014). A Systematic Review and Meta-Analysis of Total Thyroidectomy versus Bilateral Subtotal Thyroidectomy for Graves' Disease. Surgery 155, 529-540. doi:10.1016/j.surg.2013.10.017

Ferrari, S. M., Fallahi, P., Antonelli, A., and Benvenga, S. (2017). Environmental Issues in Thyroid Diseases. Front. Endocrinol. (Lausanne) 8, 50. doi:10.3389/ fendo. 2017.00050

Ferrari, S. M., Fallahi, P., Vita, R., Antonelli, A., and Benvenga, S. (2015). Peroxisome Proliferator-Activated Receptor-Гin Thyroid Autoimmunity. PPAR Res. 2015, 1-8. doi:10.1155/2015/232818

Galetta, F., Franzoni, F., Fallahi, P., Tocchini, L., Braccini, L., Santoro, G., et al. (2008). Changes in Heart Rate Variability and QT Dispersion in Patients with Overt Hypothyroidism. Eur. J. Endocrinol. 158, 85-90. doi:10.1530/EJE-070357

Harris, A., and Al Mushref, M. (2021). Graves' Thyrotoxicosis Following SARSCoV-2 Infection. AACE Clin. Case Rep. 7, 14-16. doi:10.1016/ j.aace.2020.12.005

Jiménez-Blanco, S., Pla-Peris, B., and Marazuela, M. (2021). COVID-19: a Cause of Recurrent Graves' Hyperthyroidism? J. Endocrinol. Invest. 44, 387-388. doi:10.1007/s40618-020-01440-0

Kahaly, G. J. (2020). Management of Graves Thyroidal and Extrathyroidal Disease: An Update. J. Clin. Endocrinol. Metab. 105, 3704-3720. doi:10.1210/clinem/ dgaa646

Lanzolla, G., Marcocci, C., and Marinò, M. (2021). Graves' Disease and Graves' Orbitopathy Following COVID-19. J. Endocrinol. Invest. 44, 2011-2012. doi:10.1007/s40618-021-01576-7

Leite, A. C., Pedro, A. B., and Romaldini, J. H. (2011). Influence of Methimazole and Radioactive Iodine Treatment in the Serum Levels of the Chemokine CXCL10 in Hyperthyroid Patients with Graves' Disease. Horm. Metab. Res. 43, 194-199. doi:10.1055/s-0031-1271620 
Lerner, A., Jeremias, P., and Matthias, T. (2015). The World Incidence and Prevalence of Autoimmune Diseases Is Increasing. Ijcd 3, 151-155. doi:10.12691/ijcd-3-4-8

Markham, A. (2020). Teprotumumab: First Approval. Drugs 80, 509-512. doi:10.1007/s40265-020-01287-y

Mateu-Salat, M., Urgell, E., and Chico, A. (2020). SARS-COV-2 as a Trigger for Autoimmune Disease: Report of Two Cases of Graves' Disease after COVID-19. J. Endocrinol. Invest. 43, 1527-1528. doi:10.1007/s40618-02001366-7

Mysliwiec, J., Palyga, I., Kosciuszko, M., Kowalska, A., and Gorska, M. (2012). Circulating CXCL9 and CXCL10 as Markers of Activity of Graves' Orbitopathy during Treatment with Corticosteroids and Teleradiotherapy. Horm. Metab. Res. 44, 957-961. doi:10.1055/s-0032-1316352

Paridaens, D., van den Bosch, W. A., van der Loos, T. L., Krenning, E. P., and van Hagen, P. M. (2005). The Effect of Etanercept on Graves' Ophthalmopathy: a Pilot Study. Eye (Lond) 19, 1286-1289. doi:10.1038/sj.eye.6701768

Pearce, S. H. S., Dayan, C., Wraith, D. C., Barrell, K., Olive, N., Jansson, L., et al. (2019). Antigen-Specific Immunotherapy with Thyrotropin Receptor Peptides in Graves' Hyperthyroidism: A Phase I Study. Thyroid 29, 1003-1011. doi:10.1089/thy.2019.0036

Pérez-Moreiras, J. V., Alvarez-López, A., and Gómez, E. C. (2014). Treatment of Active Corticosteroid-Resistant Graves' Orbitopathy. Ophthalmic Plast. Reconstr. Surg. 30, 162-167. doi:10.1097/IOP.0000000000000037

Perricone, C., Versini, M., Ben-Ami, D., Gertel, S., Watad, A., Segel, M. J., et al. (2016). Smoke and Autoimmunity: The Fire behind the Disease. Autoimmun. Rev. 15, 354-374. doi:10.1016/j.autrev.2016.01.001

Pritchard, J., Han, R., Horst, N., Cruikshank, W. W., and Smith, T. J. (2003). Immunoglobulin Activation of $\mathrm{T}$ Cell Chemoattractant Expression in Fibroblasts from Patients with Graves' Disease Is Mediated through the Insulin-like Growth Factor I Receptor Pathway. J. Immunol. 170, 6348-6354. doi:10.4049/jimmunol.170.12.6348

Pritchard, J., Horst, N., Cruikshank, W., and Smith, T. J. (2002). Igs from Patients with Graves' Disease Induce the Expression of T Cell Chemoattractants in Their Fibroblasts. J. Immunol. 168, 942-950. doi:10.4049/jimmunol.168.2.942

Romagnani, P., Rotondi, M., Lazzeri, E., Lasagni, L., Francalanci, M., Buonamano, A., et al. (2002). Expression of IP-10/CXCL10 and MIG/CXCL9 in the Thyroid and Increased Levels of IP-10/CXCL10 in the Serum of Patients with RecentOnset Graves' Disease. Am. J. Pathol. 161, 195-206. doi:10.1016/S00029440(10)64171-5

Ryder, M., Wentworth, M., Algeciras-Schimnich, A., Morris, J. C., Garrity, J., Sanders, J., et al. (2021). Blocking the TSH Receptor with K1-70 in a Patient with Follicular Thyroid Cancer, Graves' Disease and Graves' Ophthalmopathy. Thyroid. doi:10.1089/thy.2021.0053

Salvi, M., Girasole, G., Pedrazzoni, M., Passeri, M., Giuliani, N., Minelli, R., et al. (1996). Increased Serum Concentrations of Interleukin-6 (IL-6) and Soluble IL6 Receptor in Patients with Graves' Disease. J. Clin. Endocrinol. Metab. 81, 2976-2979. doi:10.1210/jcem.81.8.8768861

Salvi, M., Vannucchi, G., Currò, N., Campi, I., Covelli, D., Dazzi, D., et al. (2015). Efficacy of B-Cell Targeted Therapy with Rituximab in Patients with Active Moderate to Severe Graves' Orbitopathy: a Randomized Controlled Study. J. Clin. Endocrinol. Metab. 100, 422-431. doi:10.1210/jc.2014-3014

Salvi, M., Vannucchi, G., Currò, N., Introna, M., Rossi, S., Bonara, P., et al. (2012). Small Dose of Rituximab for Graves Orbitopathy: New Insights into the Mechanism of Action. Arch. Ophthalmol. 130, 122-124. doi:10.1001/ archopthalmol.2011.1215

Sánchez-Bilbao, L., Martínez-López, D., Revenga, M., López-Vázquez, Á., LópezVázquez, Á., Valls-Pascual, E., et al. (2020). Anti-IL-6 Receptor Tocilizumab in Refractory Graves' Orbitopathy: National Multicenter Observational Study of 48 Patients. J. Clin. Med. 9, 2816. doi:10.3390/jcm9092816

Schall, T. J., Jongstra, J., Dyer, B. J., Jorgensen, J., Clayberger, C., Davis, M. M., et al. (1988). A Human T Cell-specific Molecule Is a Member of a New Gene Family. J. Immunol. 141, 1018-1025.

Scott, L. J. (2014). Etanercept: a Review of its Use in Autoimmune Inflammatory Diseases. Drugs 74, 1379-1410. doi:10.1007/s40265-014-0258-9
Shoenfeld, Y., Ehrenfeld, M., and Perry, O. (2019). The Kaleidoscope of Autoimmunity - from Genes to Microbiome. Clin. Immunol. 199, 1-4. doi:10.1016/j.clim.2018.12.003

Skov, J., Eriksson, D., Kuja-Halkola, R., Höijer, J., Gudbjörnsdottir, S., Svensson, A. M., et al. (2020). Co-aggregation and Heritability of Organ-specific Autoimmunity: a Population-Based Twin Study. Eur. J. Endocrinol. 182, 473-480. doi:10.1530/EJE-20-0049

Smith, T. J., and Hegedüs, L. (2016). Graves' Disease. N. Engl. J. Med. 375, 1552-1565. doi:10.1056/NEJMra1510030

Smith, T. J. (2021). Insulin-Like Growth Factor Pathway and the Thyroid. Front. Endocrinol. (Lausanne) 12, 653627. doi:10.3389/fendo.2021.653627

Smith, T. J., Kahaly, G. J., Ezra, D. G., Fleming, J. C., Dailey, R. A., Tang, R. A., et al. (2017). Teprotumumab for Thyroid-Associated Ophthalmopathy. N. Engl. J. Med. 376, 1748-1761. doi:10.1056/NEJMoa1614949

Smith, T. J. (2019). The Insulin-like Growth Factor-I Receptor and its Role in Thyroid-Associated Ophthalmopathy. Eye (Lond) 33, 200-205. doi:10.1038/ s41433-018-0265-2

Stan, M. N., Garrity, J. A., Carranza Leon, B. G., Prabin, T., Bradley, E. A., and Bahn, R. S. (2015). Randomized Controlled Trial of Rituximab in Patients with Graves' Orbitopathy. J. Clin. Endocrinol. Metab. 100, 432-441. doi:10.1210/ jc.2014-2572

Tomer, Y., Ban, Y., Concepcion, E., Barbesino, G., Villanueva, R., Greenberg, D. A., et al. (2003). Common and Unique Susceptibility Loci in Graves and Hashimoto Diseases: Results of Whole-Genome Screening in a Data Set of 102 Multiplex Families. Am. J. Hum. Genet. 73, 736-747. doi:10.1086/378588

Turcu, A. F., Kumar, S., Neumann, S., Coenen, M., Iyer, S., Chiriboga, P., et al. (2013). A Small Molecule Antagonist Inhibits Thyrotropin Receptor AntibodyInduced Orbital Fibroblast Functions Involved in the Pathogenesis of Graves Ophthalmopathy. J. Clin. Endocrinol. Metab. 98, 2153-2159. doi:10.1210/ jc.2013-1149

Vejrazkova, D., Vcelak, J., Vaclavikova, E., Vankova, M., Zajickova, K., Duskova, M., et al. (2018). Genetic Predictors of the Development and Recurrence of Graves' Disease. Physiol. Res. 67 (Suppl. 3), S431-S439. doi:10.33549/ physiolres. 934018

Wémeau, J. L., Klein, M., Sadoul, J. L., Briet, C., and Vélayoudom-Céphise, F. L. (2018). Graves' Disease: Introduction, Epidemiology, Endogenous and Environmental Pathogenic Factors. Ann. Endocrinol. (Paris) 79, 599-607. doi:10.1016/j.ando.2018.09.002

Wiersinga, W. M., and Bartalena, L. (2002). Epidemiology and Prevention of Graves' Ophthalmopathy. Thyroid 12, 855-860. doi:10.1089/ 105072502761016476

Yokota, S., Imagawa, T., Mori, M., Miyamae, T., Aihara, Y., Takei, S., et al. (2008). Efficacy and Safety of Tocilizumab in Patients with Systemic-Onset Juvenile Idiopathic Arthritis: a Randomised, Double-Blind, Placebo-Controlled, Withdrawal Phase III Trial. Lancet 371, 998-1006. doi:10.1016/S01406736(08)60454-7

Conflict of Interest: The authors declare that the research was conducted in the absence of any commercial or financial relationships that could be construed as a potential conflict of interest.

Publisher's Note: All claims expressed in this article are solely those of the authors and do not necessarily represent those of their affiliated organizations, or those of the publisher, the editors and the reviewers. Any product that may be evaluated in this article, or claim that may be made by its manufacturer, is not guaranteed or endorsed by the publisher.

Copyright (๑ 2021 Elia, Fallahi, Ragusa, Paparo, Mazzi, Benvenga, Antonelli and Ferrari. This is an open-access article distributed under the terms of the Creative Commons Attribution License (CC BY). The use, distribution or reproduction in other forums is permitted, provided the original author(s) and the copyright owner(s) are credited and that the original publication in this journal is cited, in accordance with accepted academic practice. No use, distribution or reproduction is permitted which does not comply with these terms. 